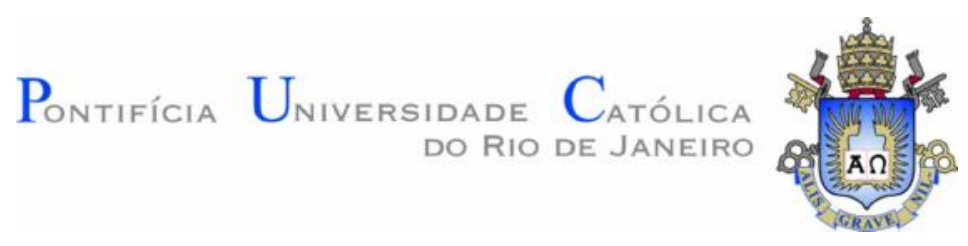

Edgar Hernán Cando Narváez

\title{
Escoamento Permanente De Um Fluido Tixotrópico Através de Expansões-Contrações Abruptas Axissimétricas
}

Dissertação de Mestrado

Dissertação apresentada como requisito parcial para obtenção do título de Mestre pelo Programa de PósGraduação em Engenharia Mecânica da PUC - Rio.

Orientador: Prof. Paulo Roberto de Souza Mendes 


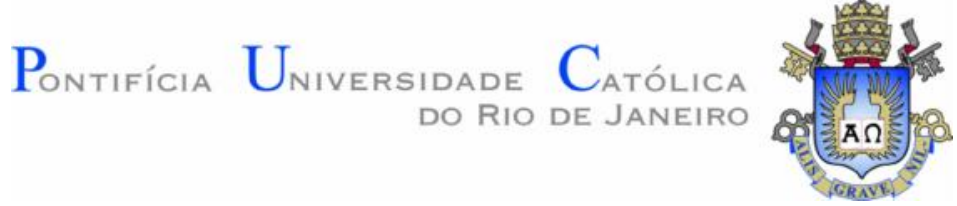

Edgar Hernán Cando Narváez

\section{Escoamento Permanente De Um Fluido Tixotrópico Através de Expansões-Contrações Abruptas}

Axissimétricas

Dissertação apresentada como requisito parcial para obtenção do título de Mestre pelo Programa de PósGraduação em Engenharia Mecânica da PUC-Rio. Aprovada pela Comissão Examinadora abaixo assinada.

Prof. Paulo Roberto de Souza Mendes Departamento de Engenharia Mecânica - PUC-Rio

Prof. Monica Feijo Naccache

Departamento de Engenharia Mecânica - PUC-Rio

Prof. Roney Leon Thompson

Departamento de Engenharia Mecânica - Universidade Federal

Fluminense

Prof. José Eugenio Leal

Coordenador Setorial do Centro Técnico Científico - PUC-Rio

Rio de Janeiro

5 de Março de 2012 
Todos os direitos reservados. É proibida a reprodução total ou parcial do trabalho sem autorização da universidade, do autor e do orientador.

\section{Edgar Hernán Cando Narváez}

Graduou-se em Engenharia Mecânica na Escuela Politécnica Nacional - EPN (Quito, Equador) em 2009.

Ficha Catalográfica

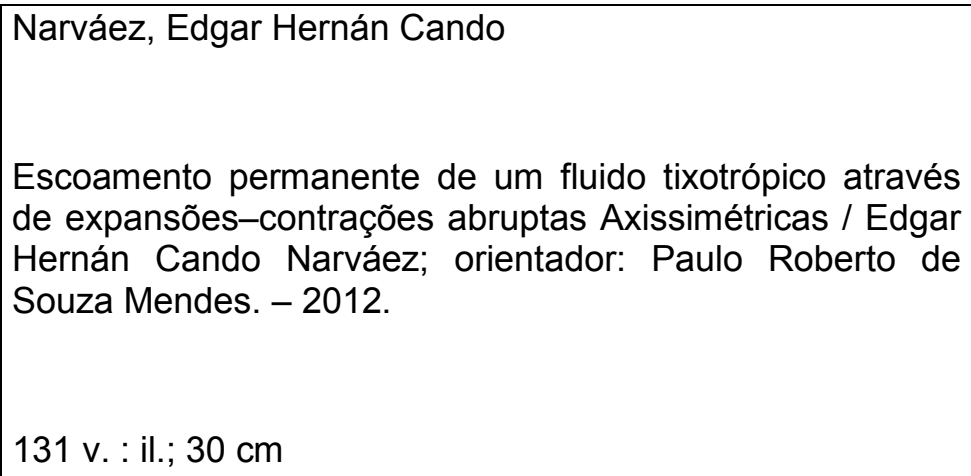
de expansões-contrações abruptas Axissimétricas / Edgar Hernán Cando Narváez; orientador: Paulo Roberto de Souza Mendes. - 2012.

131 v. : il.; $30 \mathrm{~cm}$

1. Dissertação (mestrado)-Pontifícia Universidade Católica do Rio de Janeiro, Departamento de Engenharia Mecânica, 2012.

Incluí bibliografia.

1. Engenharia mecânica - Teses. 2. Tixotropia. 3. Expansão-contração. 4. Tensão limite de escoamento. 5. Visualização de escoamentos com laser. I. Mendes, Paulo Roberto de Souza. II. Pontifícia Universidade Católica do Rio de Janeiro. Departamento de Engenharia Mecânica. III. Título.

CDD: 621 
"Quando uma pessoa deseja muito alguma coisa, o universo conspira a seu favor. Basta interpretar uma linguagem que está além das palavras e revela aquilo que os olhos não podem ver" Paulo Coelho. 


\section{Agradecimentos}

A Deus pela sua companhia e a graça de ter me permitido concluir este trabalho.

A minha família, minha mãe Marcia Noemi Narváez Duran pelo amor, apoio, compreensão e ensinamentos entregados ao longo de toda minha vida, onde nunca faltou em nenhum momento seja grande ou pequeno. Meu pai Hernán Rodrigo Cando Pacheco por ser meu melhor amigo e suporte em todo momento. Meus irmãos Eduardo e Efren por ser minha constante motivação; e todos meus tios e primos que estiveram preocupados por mim ao longos deste tempo.A minha amada Lorena por sempre estar ao meu lado com seu amor único nela, compreensão e sonhos de vida.

Ao meu orientador, professor Paulo Roberto de Souza Mendes, pelos ensinamentos, dedicação, apoio e confiança em mim prestada.

Ao Grupo de Reologia da Pontifica Universidade Católica de Rio de Janeiro em especial a Priscilla Varges pela constante ajuda, paciência e apoio entregado em todo momento durante o desenvolvimento da pesquisa.

Ao Departamento de Engenharia Mecânica da Pontifícia Universidade Católica de Rio de Janeiro.

Agradecimentos em particular para os órgãos de fomento à pesquisa $\mathrm{CNPq}$, CAPES e principalmente FAPERJ, pelo apoio financeiro fornecido, sem o qual este trabalho não teria ser possível.

Aos membros da banca examinadora, que aceitaram revisar o trabalho e contribuíram com valiosas observações. 


\section{Resumo}

Narváez, Edgar Hernán Cando; Souza Mendes, Paulo. Escoamento Permanente De Um Fluido Tixotrópico Através de ExpansõesContrações Abruptas Axissimétricas. Rio de Janeiro, 2012. 131p. Dissertação de Mestrado - Departamento de Engenharia Mecânica, Pontifícia Universidade Católica do Rio de Janeiro.

Neste trabalho estuda-se experimentalmente o comportamento dos fluidos tixotrópicos. Conseguiu-se imagens de fluidos tixotrópicos escoando através de uma expansão seguida de uma contração, ambas abruptas e axissimétricas. A existência de uma "superfície de cedêndia" produto da fronteira entre o fluido que escoa e o que não escoa, que estende-se da entrada da expansão até a saída da contração, é o principal fenômeno a ser estudado. O número de Re, o tempo para vazão mássica constante e as dimensões da geometria são as variáveis do experimento. Para o desenvolvimento do experimento utilizou-se uma dispersão de laponita $\mathrm{RD}$, que é um fluido transparente com boas características tixotrópicas. Este fluido foi caracterizado reologicamente através da obtenção da flow curve e de testes oscilatórios Strain sweep e frequency sweep. A caracterização reológica apresentou alguns problemas com respeito ao envelhecimento, tipo de geometria e reômetro utilizado, além da falta de repetitividade para baixas taxas de deformação devido ao não pré-cisalhamento da amostra. Todos os problemas foram analisados para dar solução. Os resultados mostraram que a laponita RD é um fluido tixotrópico com característica elástica na região viscoelástica linear. O escoamento do fluido através de uma expansão seguida de uma contração axissimétrica apresentou três tipos de forma que dependem da relação L/D, D/d e o número de Reynolds: escoamento de regime tubular, escoamento de regime tubular expandido e escoamento de regime descontinuo.

\section{Palavras-chave}

Tixotropia; expansão-contração; tensão limite de escoamento; visualização de escoamentos com laser. 


\section{Abstract}

Narváez, Edgar Hernán Cando; Souza Mendes, Paulo (Advisor). Flow of thixotropic fluid through axisymmetric expansions-contractions. Rio de Janeiro, 2012. 131p. MSc. Dissertation - Departamento de Engenharia Mecânica, Pontifícia Universidade Católica do Rio de Janeiro.

The behavior of thixotropics fluids through axisymmetrics expansionscontractions is studied in this research. The main objective is to visualize the "shear banding" in the flow of a thixotropic fluid, which is the interface between a flowing fluid and a non-flowing fluid. This research uses an experimental procedure to visualize the "shear banding" within the fluid. The variables used in the experimental procedure were: steady flow rate time, flow rate and geometric characteristics. A dispersion of laponite RD was utilized as the experimental fluid due to its thixotropic and transparent properties. The flow curve, strain sweep and frequency sweep of the fluid were used to obtain the rheology characterization. The rheology characterization showed lack of repeatability due to several factors, such as: aging, type of rheometers, geometry used in the rheometers and lack pre shearing of the sample. All of these issues have been analyzed to give solutions. Finally, the results showed that laponita RD is a thixotropic fluid and elastic in the viscoelastic region. Also that the flow of the fluid through the axisymmetrics expansion-contraction device has three types of behaviors: constant flow area, variable flow area with an expansion in the middle zone and variable flow area with layers of different velocities.

\section{Keywords}

Thixotropy; expansions-contractions; Yield stress; visualization of flow with laser. 


\section{Sumário}

1 Introdução 19

1.1. Motivação 20

1.2. Revisão Bibliográfica 21

1.3. Objetivos 30

1.4. Escopo 31

2 Aparato Experimental 32

2.1. Suportes 32

2.1.1. Sistema Hidráulico 33

2.1.2. Mecanismo de Visualização 34

2.2. Componentes do Aparato Experimental 36

2.2.1. Reservatório de armazenagem do fluido de trabalho 36

2.2.2. Bomba 38

2.2.3. Seção de expansão-contração 39

2.2.4. Reservatório da seção de teste 41

2.2.5. Laser 42

2.2.6. Espelho e suporte 43

2.2.7. Câmera fotográfica 44

2.2.8. Partículas para visualização 44

2.2.9. Balança 45

2.3. Montagem da Bancada 45

2.4. Procedimento Experimental 47

2.4.1. Preenchido do fluido de trabalho na bancada 49

2.4.2. Calibração do sistema laser $\quad 50$

3 Reologia do fluido $\quad 52$

3.1. O Fluido 52

3.1.1. Envelhecimento da laponita em regime permanente 54

3.1.2. Influência dos parâmetros de formação de suspensões de laponita 55

3.1.3. Resumo dos parâmetros do fluido de trabalho, laponita RD, para o $\begin{array}{lr}\text { estudo } & 58\end{array}$ 
3.1.4. Protocolo de preparação da laponita RD

3.2. Procedimento de caracterização do comportamento tixotrópico 59

3.3. Desenvolvimento dos testes de caracterização reológica 61

3.3.1. Testes oscilatórios 61

3.3.2. Testes em condições de regime permanente $\quad 62$

4 Resultados e discussão $\quad 65$

4.1. Caracterização Reológica 65

4.1.1. Testes Oscilatórios $\quad 65$

4.1.2. Testes em condições de regime permanente $\quad 67$

4.1.3. Comparação dos resultados dos testes oscilatórios e testes em condições de regime permanente $\quad 76$

4.2. Experimentos de visualização 78

4.2.1. Cálculo do número de Reynolds do escoamento na entrada da secção de expansão-contração

4.2.2. Parâmetro $t_{\mathrm{eq}}$ (tempo característicos de mudanças de um nível estrutural $\begin{array}{ll}\text { para outro) } & 79\end{array}$

4.2.3. Influência do número de Reynolds $\quad 80$

4.2.3.1. Análise do escoamento para números de Reynolds baixos sem efeitos de inércia em função da geometria $\quad 80$

4.2.3.2. Análise do escoamento com números de Reynolds altos com efeitos $\begin{array}{ll}\text { de inércia } & 90\end{array}$

4.2.4. Tipos de escoamento dentro da superfícies de cedêndia através das seções de expansão-contração em função do número de Reynolds, 93

4.2.5. Análise de mudanças do escoamento do fluido 96

4.2.6. Tempo que o fluido está escoando a vazão constante imposta pela bomba 98

4.2.7. Influência da relação da geometria L/D 99

4.2.8. Comparação do escoamento de dois fluidos viscoplásticos com diferentes níveis de tixotropia através da seção de expansão-contração. 100

5 Conclusões e recomendações 103

5.1. Conclusões e Sugestões 103

5.2. Sugestões para trabalhos futuros 104 
$\begin{array}{ll}\text { A.Anexo A } & 112\end{array}$

A.1. Informação adicional sobre a caracterização Reológica 112

A.1.1. Seleção da geometria para teste no reômetro 112

A.1.2. Influência da evaporação 114

A.1.3. Influência da temperatura nos testes 115

A.1.4. Influência do pré-cisalhamento e dificuldade de obtenção de dados a baixas taxas de deformação 116

A.1.5. Diferença na utilização do reômetro de taxa de deformação controlada e o reômetro com tensão de cisalhamento controlada 119

$\begin{array}{ll}\text { B.Anexo B } & 127\end{array}$

B.1. Análise de Incerteza 127

B.1.1. Cálculo da incerteza na medição de vazão mássica 128

B.1.2. Cálculo da incerteza da função viscosidade 129

B.1.3. Cálculo da incerteza do número de Reynolds 130 


\section{Lista de Figuras}

Figura 1-1: Demonstração da resposta da taxa de deformação pouco sensível ao início de uma aplicação de uma tensão constante em fluidos com alto nível de tixotropia , testadas suspensões de Bentonita imediatamente após de um pré-cisalhamento de $26 \mathrm{~Pa}$ durante $60 \mathrm{~s}[15]$.

Figura 1-2: Para tensões altas de cisalhamento algumas suspensões atingem um equilíbrio próprio da tixotropia, mas para baixos valores não. Testando suspensões de Bentonita com pré-cisalhamento de $27 \mathrm{~Pa}$ por $60 \mathrm{~s}$ com um repouso seguinte de $20 \mathrm{~s} \mathrm{[15].} 25$

Figura 1-3: Esquema da expansão-contração abrupta.

Figura 1-4: Fenômeno da superfície de cedêndia para três vazões distintas [46].

Figura 2-1: Bancada experimental.

Figura 2-2: Plano do laser para visualização.

Figura 2-3: Esquema do experimento. 36

Figura 2-4: Reservatório com tampa e termômetro. 37

Figura 2-5: Bomba de deslocamento positivo (Cole Parmer EW-07553-70). 38

Figura 2-6:Controlador da vazão da bomba. 39

Figura 2-7: Pulse dampener. 39

Figura 2-8: Expansão e contração. $\quad 40$

Figura 2-9: Vista da montagem da expansão-contração. 41

Figura 2-10: Expansão-contração e aquário montados. 42

Figura 2-11: Laser utilizado. 43

Figura 2-12: Fontes do laser. 43

Figura 2-13: O espelho e os acessórios de seu suporte. 44

Figura 2-14: Balança Gehaka utilizada para medir a vazão. 45

Figura 2-15: Posicionamento e alinhamento do laser. 46

Figura 2-16: Alinhamento do sistema de suporte do espelho. 47

Figura 2-17: Calibração da posição do laser. $\quad 51$

Figura 3-1: Estrutura "casa de cartas" do gel de laponita. 56

Figura 3-2: Procedimento de caracterização do comportamento tixotrópico [10]. 
Figura 3-3: Reômetro Ares G2 (TA instruments) do laboratório de reologia da PUC-RIO.

Figura 3-4: Reometro Physica MCR 501 do laboratório de Reologia da PUC-RIO.

Figura 4-1: Teste Strain sweep da dispersão de $2 \%$ de laponita RD.

Figura 4-2: Teste frequency sweep da dispersão de $2 \%$ de laponita RD.

Figura 4-3: Evolução de viscosidade com o tempo de uma suspensão aquosa de $2 \%$ de laponita RD, correspondente á aplicação no instante $\mathrm{t}=0$ s de uma taxa de deformação de 500 1/s. Azul: $\gamma=100$ 1/s para $\mathrm{t}<0 \mathrm{~s}$, Vermelho $\gamma=10001 / \mathrm{s}$ para $\mathrm{t}<0 \mathrm{~s}$.

Figura 4-4: Teste step change de 100 a 1000 (1/s) e step change de 1500 a 1000 (1/s) para a suspensão aquosa de $2 \%$ de laponita RD do protocolo de preparação.

Figura 4-5: Teste step change de 1000 a 500 (1/s) e step change de 100 a $500(1 / \mathrm{s})$ com pré-cisalhamento de 1500 1/s para a suspensão aquosa de $2 \%$ de laponita RD do protocolo de preparação.

Figura 4-6: Teste step change de 1000 a 100 (1/s) e step change de 1 a 100 (1/s) com pré-cisalhamento de 1500 1/s, para a suspensão aquosa de $2 \%$ de laponita RD do protocolo de preparação.

Figura 4-7: Teste step change de 100 a 1 (1/s) e step change de 0.1 a $1(1 / \mathrm{s})$ com pré-cisalhamento de 1500 1/s, para a suspensão aquosa de $2 \%$ de laponita RD do protocolo de preparação.

Figura 4-8: Testes tixotrópicos de 1500 e 1000 (1/s) de suspensões de $2 \%$ de laponita RD do protocolo de preparação.

Figura 4-9: Teste tixotrópicos de 500 e 100 (1/s) de suspensões de 2\% de laponita RD do protocolo de preparação.

Figura 4-10: Teste Step change de 100 a 9.25 1/s, 100 a 5 1/s, 100 a

3 1/s, 100 a 1.75 1/s, 100 a 1 1/s e 100 a 50 1/s para a suspensão aquosa de laponita RD, com pré-cisalhamento de 1500 1/s.

Figura 4-11: Reologia em regime permanente da laponita RD preparada. Figura 4-12: Explicação da combinação do comportamento tixotrópico e elástico em um fluido. (a) fluido puramente tixotrópico, (b) fluido puramente elástico, c) fluido com características tixotropicas e elásticas. 
Figura 4-13: Analogia mecânica do modelo proposto por Souza Mendes [47].

Figura 4-14: Imagens do escoamento da dispersão de $2 \%$ de laponita RD através de uma seção de expansão-contração ambas abruptas com a relação de dimensões $d-D-d=1-3-1$ e $L / D=0.5 / 1$ para diferentes vazões mássicas: (a) $\mathrm{m}=0.223 \mathrm{~g} / \mathrm{s}, \mathrm{Re}=0.006$, (b) $\mathrm{m}=1.244 \mathrm{~g} / \mathrm{s}, \mathrm{Re}=0.150$, (c) $\mathrm{m}=1.999 \mathrm{~g} / \mathrm{s}$, $\mathrm{Re}=0.346$, (d) $\mathrm{m}=3.147 \mathrm{~g} / \mathrm{s}, \operatorname{Re}=0.749$.

Figura 4-15: Imagens do escoamento da dispersão de $2 \%$ de laponita RD através de uma seção de expansão-contração ambas abruptas com a relação de dimensões $\mathrm{d}-\mathrm{D}-\mathrm{d}=1-3-1$, e $\mathrm{L} / \mathrm{D}=1 / 1$, para diferentes vazões mássicas: (a) $\mathrm{m}=0.801 \mathrm{~g} / \mathrm{s}, \mathrm{Re}=0.067$, (b) $\mathrm{m}=1.010 \mathrm{~g} / \mathrm{s}, \mathrm{Re}=0.103$, (c) $\mathrm{m}=1.689 \mathrm{~g} / \mathrm{s}$, $\mathrm{Re}=0.258$ (d) $\mathrm{m}=1.940 \mathrm{~g} / \mathrm{s}, \mathrm{Re}=0.495$.

Figura 4-16: Imagem do escoamento da dispersão de $2 \%$ de laponita RD através de uma seção de expansão-contração ambas abruptas com a relação de dimensões d-D-d=1-3-1, e $\mathrm{L} / \mathrm{D}=1.5 / 1$, para $\mathrm{m}=0.610 \mathrm{~g} / \mathrm{s}$, $\operatorname{Re}=0.04$.

Figura 4-17: Imagem do escoamento da dispersão de $2 \%$ de laponita RD através de uma seção de expansão-contração ambas abruptas com a relação de dimensões d-D-d=1-3-1, e $\mathrm{L} / \mathrm{D}=1.5 / 1$, para $\mathrm{m}=1.016 \mathrm{~g} / \mathrm{s}, \mathrm{Re}=0.104$.

Figura 4-18: Imagem do escoamento da dispersão de $2 \%$ de laponita RD através de uma seção de expansão-contração ambas abruptas com a relação de dimensões d-D-d=1-3-1, e $\mathrm{L} / \mathrm{D}=1.5 / 1$, para $\mathrm{m}=1.223 \mathrm{~g} / \mathrm{s}, \mathrm{Re}=0.145$.

Figura 4-19: Imagem do escoamento da dispersão de $2 \%$ de laponita RD através de uma seção de expansão-contração ambas abruptas com a relação de dimensões d-D-d=1-3-1, e $\mathrm{L} / \mathrm{D}=1.5 / 1$, para $\mathrm{m}=1.649 \mathrm{~g} / \mathrm{s}, \mathrm{Re}=0.240$.

Figura 4-20: Imagens do escoamento da dispersão de $2 \%$ de laponita RD através de uma seção de expansão-contração ambas abruptas com a relação de dimensões $d-D-d=1-5-1$, e $L / D=0.5 / 1$, para diferentes vazões mássicas: (a) $\mathrm{m}=0.614 \mathrm{~g} / \mathrm{s}, \operatorname{Re}=0.041$, (b) $\mathrm{m}=1.173 \mathrm{~g} / \mathrm{s}, \mathrm{Re}=0.135$, (c) $\mathrm{m}=1.583 \mathrm{~g} / \mathrm{s}$, $\mathrm{Re}=0.230$, (d) $\mathrm{m}=1.960 \mathrm{~g} / \mathrm{s}, \mathrm{Re}=0.500$.

Figura 4-21: Imagens do escoamento da dispersão de $2 \%$ de laponita RD através de uma seção de expansão-contração ambas abruptas com a relação de dimensões $\mathrm{d}-\mathrm{D}-\mathrm{d}=1-5-1$, e $\mathrm{L} / \mathrm{D}=1 / 1$, para diferentes vazões mássicas: 
(a) $\mathrm{m}=0.300 \mathrm{~g} / \mathrm{s}, \mathrm{Re}=0.010$, (b) $\mathrm{m}=0.699 \mathrm{~g} / \mathrm{s}, \mathrm{Re}=0.052$, (c) $\mathrm{m}=0.896 \mathrm{~g} / \mathrm{s}$, $\operatorname{Re}=0.083, \quad(d) \mathrm{m}=1.235 \mathrm{~g} / \mathrm{s}, \operatorname{Re}=0.148$.

Figura 4-22: Imagem do escoamento da dispersão de $2 \%$ de laponita RD através de uma seção de expansão-contração ambas abruptas com a relação de dimensões d-D-d=1-5-1, e L/D=1.5/1, para $\mathrm{m}=0.520 \mathrm{~g} / \mathrm{s}, \mathrm{Re}=0.030$. Figura 4-23: Imagem do escoamento da dispersão de $2 \%$ de laponita RD através de uma seção de expansão-contração ambas abruptas com a relação de dimensões d-D-d=1-5-1, e $\mathrm{L} / \mathrm{D}=1.5 / 1$, para $\mathrm{m}=0.672 \mathrm{~g} / \mathrm{s}, \mathrm{Re}=0.048$.

Figura 4-24: Imagem do escoamento da dispersão de $2 \%$ de laponita RD através de uma seção de expansão-contração ambas abruptas com a relação de dimensões d-D-d=1-5-1, e L/D=1.5/1, para $\mathrm{m}=0.987 \mathrm{~g} / \mathrm{s}, \mathrm{Re}=0.098$.

Figura 4-25: Imagem do escoamento da dispersão de $2 \%$ de laponita RD através de uma seção de expansão-contração ambas abruptas com a relação de dimensões d-D-d=1-5-1, e L/D=1.5/1, para $\mathrm{m}=1.943 \mathrm{~g} / \mathrm{s}, \mathrm{Re}=0.330$.

Figura 4-26: Imagens do escoamento da dispersão de $2 \%$ de laponita RD através de uma seção de expansão-contração ambas abruptas com a relação de dimensões d-D-d=1-5-1. (a) $\mathrm{L} / \mathrm{D}=0.5 / 1, \mathrm{~m}=4.410 \mathrm{~g} / \mathrm{s}, \mathrm{Re}=1.307$, (b) $\mathrm{L} / \mathrm{D}=1 / 1, \mathrm{~m}=3.690 \mathrm{~g} / \mathrm{s}, \mathrm{Re}=0.976$, (c) $\mathrm{L} / \mathrm{D}=1.5 / 1, \mathrm{~m}=8.080 \mathrm{~g} / \mathrm{s}, \quad \mathrm{Re}=$ 3.410 .

Figura 4-27: Imagens do escoamento da dispersão de $2 \%$ de laponita RD através de uma seção de expansão-contração ambas abruptas com a relação de dimensões $\mathrm{d}-\mathrm{D}-\mathrm{d}=1-5-1$, e $\mathrm{L} / \mathrm{D}=0.5 / 1$, para vazão mássicas de $\mathrm{m}=4.410 \mathrm{~g} / \mathrm{s}, \mathrm{Re}=1.307$.(a) tempo de escoamento $\mathrm{t}=0$, (b) tempo de escoamento $\mathrm{t}=300 \mathrm{~s}$.

Figura 4-28: Imagens do escoamento da dispersão de $2 \%$ de laponita RD através de uma seção de expansão-contração ambas abruptas com a relação de dimensões $d-D-d=1-5-1$, e $L / D=0.5 / 1$, para diferentes vazões mássicas com $\operatorname{Re}$ altos. (a) $\mathrm{m}=4.410 \mathrm{~g} / \mathrm{s}, \mathrm{Re}=1.307$, (b) $\mathrm{m}=5,600 \mathrm{~g} / \mathrm{s}, \mathrm{Re}=1.920$.

Figura 4-29: Mapa dos tipos de escoamento do fluido tixotrópico, dispersão de $2 \%$ de laponita RD, através de uma expansão- contração abrupta e assimétrica.

Figura 4-30: Comparação do escoamento da dispersão de $2 \%$ de laponita RD através de uma seção de expansão-contração ambas abruptas com a relação de 
dimensões d-D-d=1-5-1, e $\mathrm{L} / \mathrm{D}=1 / 1$, quando o primeiro escoamento é muito mas alto que o segundo. Primeiro (a) $\mathrm{m}=3.690 \mathrm{~g} / \mathrm{s}, \mathrm{Re}=0.970$,

(b) $\mathrm{m}=0.860 \mathrm{~g} / \mathrm{s}, \mathrm{Re}=0.08$.

Figura 4-31: Comparação do escoamento da dispersão de $2 \%$ de laponita RD através de uma seção de expansão-contração ambas abruptas com a relação de dimensões $d-D-d=1-5-1$, e $L / D=1.5 / 1$, quando o primeiro escoamento é muito mas alto que o segundo. Primeiro (a) $\mathrm{m}=3.431 \mathrm{~g} / \mathrm{s}, \mathrm{Re}=0.865$,

(b) $\mathrm{m}=0.260 \mathrm{~g} / \mathrm{s}, \mathrm{Re}=0.008$.

Figura 4-32: Imagens do escoamento da dispersão de $2 \%$ de laponita RD através de uma seção de expansão-contração ambas abruptas com a relação de dimensões d-D-d=1-5-1, e $\mathrm{L} / \mathrm{D}=1 / 1$ para vazão mássica de $\mathrm{m}=0.699 \mathrm{~g} / \mathrm{s}$, $\mathrm{Re}=0.052$ com uma vazão anterior inferior.(a) tempo de escoamento $\mathrm{t}<100$ segundos, (b) tempo de escoamento $t>100$ segundos.

Figura 4-33: Comparação do escoamento da dispersão de $2 \%$ de laponita RD através de uma seção de expansão-contração ambas abruptas com a relação de dimensões d-D-d=1-5-1 variando $\mathrm{L} / \mathrm{D}$. (a) $\mathrm{L} / \mathrm{D}=0.5, \mathrm{~m}=0.614 \mathrm{~g} / \mathrm{s}$, $\mathrm{Re}=0.041$, (b) $\mathrm{L} / \mathrm{D}=1, \mathrm{~m}=0.300 \mathrm{~g} / \mathrm{s}, \mathrm{Re}=0.010$, (c) $\mathrm{L} / \mathrm{D}=1.5$, $\mathrm{m}=0.520 \mathrm{~g} / \mathrm{s}, \mathrm{Re}=0.030$.

Figura 4-34: Comparação do escoamento de dois fluidos viscoplásticos, na geometria $d-D-d=1-5-1, L / D=1 / 0.5$, com diferente nível de tixotropia. (a) Laponita Rd 2\%, $\gamma=16.65$ (1/s) $n=0.8752$ (Pa.s), (b) Carbopol 0.11\%, $\gamma=$ 20.17(1/s) $n=1.450$ (Pa.s) [46], (c) Laponita Rd 2\%, $\gamma=102.01$ (1/s) $n=0.2732$ (Pa.s), (d) Carbopol 0.11\%, $\gamma=124.80(1 / \mathrm{s}) n=0.4161$ (Pa.s) [46].

Figura A-1: Teste flow curve de 1000 a 100 (1/s) utilizando a geometria cone-placa

Figura A-2: Teste flow curve de 1000 a 100 (1/s) utilizando a geometria Couette.

Figura A-3: Testes tixotrópico a 0.01 (1/s) com a geometria de discos de placas , utilizando tampa, que apresenta a influencia da evaporação nos teste.

Figura A-4: Testes tixotrópicos a 500 1/s com um pré-cisalhamento de $250 \mathrm{~s}$, a $25^{\circ} \mathrm{C}$ e $20{ }^{\circ} \mathrm{C}$. 
Figura A-5: Dificuldade de toma de dados para baixas taxas de deformação e falta de repetitividade.

Figura A-6: (a) Testes de step change de 1500 a 1 (1/s),

(b) ampliação da região com taxa de deformação 1 (1/s).

Figura A-7: Diferença nas flow curve utilizando o reômetro ARES G2

(taxa de deformação controlada) e o PAAR Physica MCR 501

(tensão de cisalhamento controlada).

Figura A-8: Teste step change de 100 a 500 (1/s) e 500 a 100 (1/s), cinco dias após a preparação da suspensão de $2 \%$ de laponita RD.

Figura A-9: Teste step change de 100 a 500 (1/s) e 500 a 100 (1/s), seis dias após a preparação da suspensão de $2 \%$ de laponita RD.

Figura A-10: Teste step change de 100 a 500 (1/s), sete após a preparação da suspensão de $2 \%$ de laponita RD

Figura A-11: Teste step change de 100 a 500 (1/s), oito dias após a preparação da suspensão de $2 \%$ de laponita RD

Figura A-12: Comparação dos testes step-change de 100 a 500 (1/s), do sétimo e oitavo dia após a preparação da suspensão de $2 \%$ de laponita $\mathrm{RD}$

Figura A-13: Dispersão de laponita, aquecida por 2 dias após a preparação Figura A-14: Teste step change de 500 a 100 (1/s), aquecida 2 dias após a preparação da dispersão de laponita e ao testada ao quarto e quinto dia.

Figura B-1: Incerteza na medição de vazão mássica 


\section{Lista de tabelas}

Tabela 2-1: Dimensões dos tubos de acrílico da seção de expansão-contração. 40

Tabela 4-1: Características da suspensão de 2\% de laponita RD preparada. $\quad 75$

Tabela 4-2:Tabela dos tipos de perfis de escoamento através das seções de expansão- contração. 